\title{
Anesthetic Care of a Patient With Bernard-Soulier Syndrome for Posterior Spinal Fusion
}

\author{
Sushmitha S. Boppana ${ }^{\mathrm{a}}$, Brian Hall ${ }^{\mathrm{b}}$, Ashley Beaujon ${ }^{\mathrm{c}}$, \\ Dmitry Tumin ${ }^{\text {b, d }}$, Joseph D. Tobias ${ }^{\text {b, d, e, f }}$
}

\begin{abstract}
Bernard-Soulier syndrome (BSS), a rare autosomal recessive disorder first identified in 1948, is characterized by excessive and prolonged bleeding due to thrombocytopenia and platelet dysfunction with increased platelet size and deformability. The primary defect in BSS involves the glycoprotein (GP) Ib-IX-V complex, which is important in initiating platelet aggregation and thrombosis after vascular injury by facilitating the adhesion of platelets to von Willebrand factor. The coagulation defect in BSS can lead to significant bleeding during traumatic injury or surgical intervention. We present a 17 -year-old adolescent with BSS who presented for posterior spinal fusion for idiopathic scoliosis. Previous reports of perioperative care of patients with BSS are reviewed and options for anesthetic care including perioperative control of the coagulation defect are presented.
\end{abstract}

Keywords: Bernard-Soulier syndrome; Coagulation; Posterior spinal fusion; Platelet function

\section{Introduction}

Bernard-Soulier syndrome (BSS), a rare autosomal recessive disorder, was first identified in 1948 [1]. It is characterized by excessive and prolonged bleeding due to thrombocytopenia and platelet dysfunction with increased platelet size and deformability [2]. Patients with BSS have a defect in or complete lack of the key glycoprotein (GP) Ib-IX-V complex, normally

Manuscript submitted August 13, 2018, accepted September 4, 2018

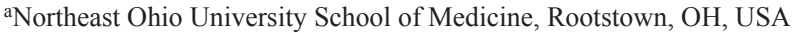
bepartment of Anesthesiology \& Pain Medicine, Nationwide Children's Hospital, Columbus, OH, USA

'Otterbein School of Nurse Anesthesia, Columbus, OH, USA

dDepartment of Pediatrics, Nationwide Children's Hospital and the Ohio State University, Columbus, OH, USA

eDepartment of Anesthesiology \& Pain Medicine, The Ohio State University College of Medicine, Columbus, OH, USA

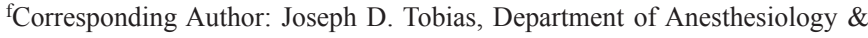
Pain Medicine, Nationwide Children's Hospital, 700 Children's Drive, Columbus, OH 43205, USA. Email: Joseph.Tobias@Nationwidechildrens.org

doi: https://doi.org/10.14740/jmc3142w found on the surface of platelets. The GPIb-IX-V is important in initiating platelet aggregation and thrombosis after vascular injury by facilitating the adhesion of platelets to von Willebrand Factor (vWF), which is exposed on the vascular subendothelium after blood vessel injury. The binding of GPIb-IX-V to $\mathrm{vWF}$ results in a decrease in velocity of platelets as they move past damaged vessel walls and is critical in the process of initiating thrombosis to repair damaged vasculature $[2,3]$. In addition to mediating adhesion of platelets to the blood vessel wall at sites of vascular injury by binding vWF, the complex facilitates platelet activation at low concentrations of thrombin, which may have roles in the platelet formation and turnover given that BSS is associated with decreased number and abnormal size. The lack of or defect in the functioning of GPIb-IX-V results in a compromised ability to initiate platelet adhesion and aggregation resulting in a bleeding diathesis [4]. Diagnosis is confirmed by platelet function testing with lack of platelet aggregation in response to ristocetin. We present a 17-year-old adolescent with BSS who presented for posterior spinal fusion for idiopathic scoliosis. Previous reports of perioperative care of patients with BSS are reviewed and options for anesthetic care including correction of the coagulation defect are presented.

\section{Case Report}

Institutional Review Board approval is not required at $\mathrm{Na}-$ tionwide Children's Hospital (Columbus, OH, USA) for the presentation of single case reports. The patient was a 17-yearold, $70.9 \mathrm{~kg}$ girl who presented for posterior spinal fusion to treat adolescent idiopathic scoliosis. Her past medical history was positive for BSS, diagnosed at 3 years of age on platelet function testing for presentation of easy bruising and excessive bleeding. She also had been diagnosed with Ehlers-Danlos syndrome, diagnosed at age of 9 years with genetic testing and idiopathic scoliosis. Due to the BSS, she has macrothrombocytopenia with a baseline platelet count in the 50,000 - 70,000/ $\mathrm{mm}^{3}$. Her past surgical history was positive for one orthodontic procedure that did not involve anesthesia and resulted in no complications. Although prior platelet transfusions had resulted in an inadequate increase in her platelet count, workup for platelet specific antibiotics was negative. Her medications at the time of surgery included tranexamic acid $(1,300$ $\mathrm{mg}$ PO TID) to control menstrual bleeding, celecoxib (100 mg 
PO BID) for menses-associated pain, an oral probiotic and a benzoyl peroxide $10 \%$ topical wash. Physical examination revealed a well-nourished and well-developed young woman in the 85th percentile for weight and 89th percentile for height. She denied any recent health concerns and her vital signs were unremarkable. Her airway examination revealed a Mallampati class I airway with a thyromental distance greater than three fingerbreadths. Her cardiorespiratory examination was unremarkable. She was eager to proceed as prior posterior spinal fusion (PSF) surgery, scheduled 3 months earlier, and was cancelled due to inadequate response to two platelet transfusions, resulting in a platelet count of $74,000 / \mathrm{mm}^{3}$, which did not meet the desired threshold of $100,000 / \mathrm{mm}^{3}$. Given the inadequate response to previous platelet transfusions, after hematology consultation, a plan was devised to further address these issues perioperatively and her surgical procedure was rescheduled. The platelet count the day prior to surgery was $72,000 /$ $\mathrm{mm}^{3}$ and she received one unit of pheresed platelets, which increased her platelet count to $78,000 / \mathrm{mm}^{3}$. Other coagulation parameters including prothrombin time (PT), partial thromboplastin time (PTT) and international normalized ration (INR) were within normal limits. Due to previous issues with a limited response to platelet transfusions, it was decided to monitor in vivo coagulation function using the rotational thromboelastogram (ROTEM $\AA$ ). The values were within normal limits except for a prolongation of the clot formation time (CFT). The patient was kept nil per os for $6 \mathrm{~h}$ before being transported to the operating room following premedication with intravenous midazolam (2 mg). Routine American Society of Anesthesiologists monitors were placed followed by anesthetic induction with propofol (200 mg), lidocaine (100 mg) and sufentanil (20 ig). Endotracheal intubation was facilitated by the administration of rocuronium $(65 \mathrm{mg})$. Following anesthetic induction, two large bore peripheral intravenous cannulas and an arterial cannula were placed. To facilitate neurophysiological monitoring per our usual protocol, maintenance anesthesia included desflurane titrated to maintain the bispetral index at $50-60$ and a sufentanil infusion adjusted from $0.1-0.3 \mathrm{ig} / \mathrm{kg} / \mathrm{h}$ to maintain the mean arterial pressure at $55-65 \mathrm{~mm} \mathrm{Hg}$ [5]. Maintenance anesthesia was supplemented by continuous infusions of lidocaine and esmolol. Prior to the start of the surgical procedure, a second unit of pheresed platelets was infused along with a single dose of recombinant factor VIIa $(1 \mathrm{mg})$. The dosing of rFVIIa was repeated every 2 - $3 \mathrm{~h}$ intraoperatively. Tranexamic acid was administered intraoperatively (loading dose of 50 $\mathrm{mg} / \mathrm{kg}$ followed by a continuous infusion of $5 \mathrm{mg} / \mathrm{kg} / \mathrm{h}$ during the procedure). The ROTEM ${ }^{\circledR}$ was followed during the perioperative course and remained at baseline except for a persistent mild elevation of the CFT. Intraoperatively, surgical hemostasis was adequate as judged by the surgical team. No allogeneic packed red blood cells were administered. Intraoperative cell savage was used with the return of $350 \mathrm{~mL}$ of salvaged and washed blood. The surgical procedure was accomplished in $4 \mathrm{~h}$ $25 \mathrm{~min}$ with an estimated blood loss of $900 \mathrm{~mL}$. At the completion of the procedure, the patient's trachea was extubated and she was transferred to the post-anesthesia care unit and then to the pediatric ICU for $24 \mathrm{~h}$. Her postoperative hemoglobin remained stable at $9-10 \mathrm{gm} / \mathrm{dL}$. On postoperative day 1 , she received one unit of pheresed platelets for a platelet count of
$56,000 / \mathrm{mm}^{3}$. Postoperatively, å-amino caproic acid (Amicar) was administered intravenously and then by mouth for a total of 10 days. No other allogeneic blood products were administered during the postoperative course and although her platelet count decreased to a nadir of $39,000 / \mathrm{mm}^{3}$, there was no clinically concerning bleeding and her ROTEM ${ }^{\circledR}$ values remained stable. Her postoperative course was unremarkable and she was discharged to the inpatient ward on postoperative day 1 and home on postoperative day 5 .

\section{Discussion}

BSS is a rare inherited disorder affecting platelet function with decreased aggregation, decreased numbers and decreased function. As such, excessive bleeding may occur spontaneously, following trauma or during surgical procedures. Although the glycoprotein defect results in the ineffective interaction of $\mathrm{vWf}$ with the vascular endothelium, the disorder cannot be corrected by the administration of $\mathrm{vWf}$ and other strategies are needed. Our case was unique in two respects including our patient's refractory state to the administration of allogeneic platelets with minimal increase in the platelet count. Despite this, work-up did not reveal platelet antibodies or other issues that might be amenable to corticosteroid therapy or plasmapheresis.

While the mainstay remains the administration of allogeneic platelets, adjunctive strategies have included the administration of antifibrinolytic agents ( $\varepsilon$-amino caproic acid or tranexamic acid), recombinant factor VIIa and 1-deamino8 -d-arginine vasopressin (DDAVP). To date, there are a limited number of reports in the literature regarding the perioperative care of pediatric patients with BSS (Table 1) [2, 6-15]. While the mainstay of therapy includes platelet transfusions, adjunctive therapies have included desmopressin, anti-fibrinolytic agents (tranexamic acid or $\varepsilon$-aminocaproic acid) or recombinant factor VIIa. Given our patient's inadequate response to platelet transfusions, we chose to use adjunctive agents to augment platelet and coagulation function including tranexamic acid and recombinant VIIa.

Tranexamic acid is a synthetic, lysine-derived antifibrinolytic agent used to slow the breakdown of thrombin and fibrin, thereby limiting blood loss in various surgical procedures and in trauma patients [16]. By reversibly blocking the five lysine binding sites on plasminogen, tranexamic acid prevents the conversion of plasminogen to plasmin and the degradation of fibrin. Tranexamic acid has been shown to effectively limit blood loss during orthopedic procedures on the spine even in patients with normal preoperative coagulation function $[17,18]$. In addition to its effects on the fibrinolytic process, tranexamic acid also improves platelet function through poorly understood and controversial mechanisms [19]. Clinical studies have demonstrated that tranexamic acid attenuates platelet aggregation defects created by antiplatelet treatment or by uremia $[20,21]$. These effects may explain its anecdotal success in patients with BSS and other platelet defects.

In addition, we chose to use recombinant factor VIIa (rFVIIa), a synthetic hemostatic agent originally used to treat bleeding in hemophilia A patients who had developed inhibitors to factor VIII $[22,23]$. Given its integral effect of coagula- 
Table 1. Reports of Perioperative Care of Patients With Bernard-Soulier Syndrome

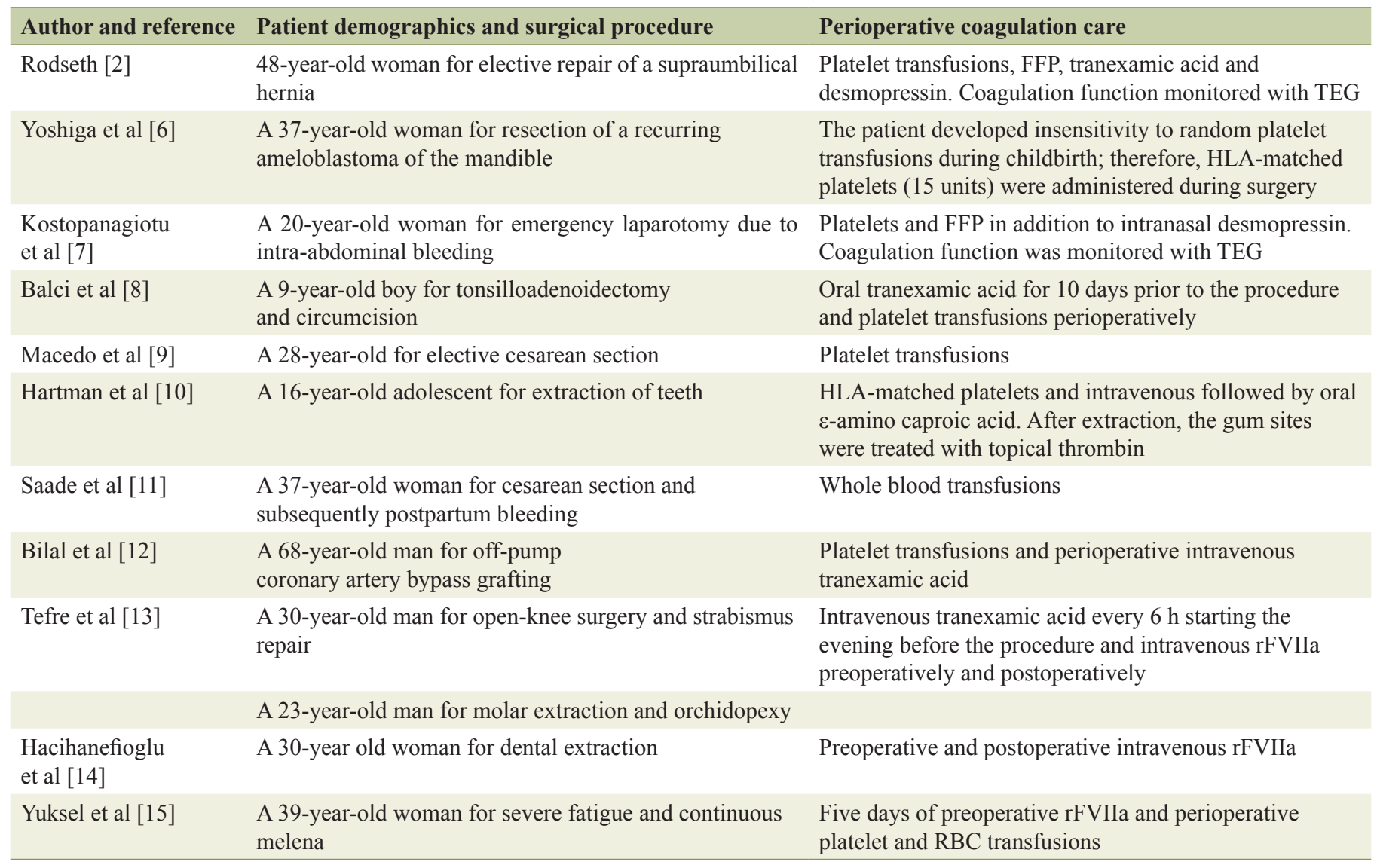

FFP: fresh frozen plasma; TEG: thromboelastogram; PPI: proton pump inhibitor; rFVIla: recombinant factor VIla; RBC: packed red blood cell.

tion function, rFVIIa has also been used to treat bleeding of various other etiologies in many clinical scenarios. Following disruption of the endothelial barrier of blood vessels, tissue factor (TF) is exposed and binds factor VIIa [24]. The complex of TF and VIIa initiates the extrinsic coagulation cascade with the activation of factor $\mathrm{X}$ to $\mathrm{Xa}$, which in association with cofactor $\mathrm{Va}$, leads to the formation of thrombin from prothrombin. The conversion of prothrombin to thrombin occurs on the surface of activated platelets, resulting in the cleavage of fibrinogen to fibrin, which is crosslinked and further recruits platelets to propagate the thrombus. Though not well understood, rFVIIa, by directly activating factor $\mathrm{X}$, is thought to help compensate for low or dysfunctional platelets by increasing the generation of thrombin and the subsequent activation of platelets are activated $[25,26]$. In addition to these proposed cellular mechanisms, rFVIIa has been shown to anecdotally be a valuable adjunct in preventing or the treatment of bleeding in patients with qualitative and quantitative platelet disorders [27].

Given that the routine laboratory parameters of coagulation function (PT and PTT) do not demonstrate the clinical interaction of the various components of the coagulation cascade or adequately demonstrate platelet function, we used the ROTEM ${ }^{\circledR}$ to evaluate and follow in vivo coagulation function in our patient. Despite the presence of persistent thrombocytopenia following platelet transfusions, coagulation function was clinically adequate to allow for completion of the surgical procedure. The ROTEM ${ }^{\circledR}$ is a modification of the thromboelastogram that identifies clot formation and strength by using an electromechanical transduction system that measures the amount of rotational force transmitted by the developing clot $[28,29]$. The system involves the suspension of ball from a rod in a rotational device. The rotation of the ball is impeded as the clot develops, thereby characterizing the patient's hemostatic ability. Clot development in vivo is dependent on the interaction of platelets with coagulation factors which can be evaluated by the pattern seen on the ROTEM ${ }^{\circledR}$. The propagation of clot and the pattern can also be followed to evaluate for clot lysis (fibrinolysis), hence evaluating the efficacy of agents. We utilized the coagulation profile provided by ROTEM ${ }^{\circledR}$ to assess our patient's qualitative response to platelet transfusions, response to adjunctive agents (tranexamic acid and rFVIIa), the risk for perioperative bleeding and transfusion needs in the perioperative period.

In summary, we present the perioperative care of an adolescent with BSS for PSF. Although platelet transfusions remain the mainstay of therapy to prevent or treat bleeding, adjunction agents including anti-fibrinolytic agents or rFVIIa have been shown to be useful. Our anecdotal experience adds to the literature suggesting the demonstration of utility of bedside point-of-care coagulation function monitoring using the 
ROTEM ${ }^{\circledR}$. With such care, even invasive surgical procedures can be accomplished with minimal sequelae related to the primary bleeding disorder.

\section{References}

1. Bernard J, Soulier JP. Sur une nouvelle variete de dystrophie thrombocytaire-hemorragipare congenitale. Sem Hop. 1948;24(Spec. No.):3217-3223.

2. Rodseth RN. The perioperative management of Bernard-Soulier syndrome: a case report and review of the role of perioperative factor VIIa. S Afr J Anesth Analg. 2010;16:37-39.

3. Andrews RK, Gardiner EE, Shen Y, Whisstock JC, Berndt MC. Glycoprotein Ib-IX-V. Int J Biochem Cell Biol. 2003;35(8):1170-1174.

4. Berndt MC, Andrews RK. Bernard-Soulier syndrome. Haematologica. 2011;96(3):355-359.

5. Martin DP, Bhalla T, Thung A, Rice J, Beebe A, Samora W, Klamar J, et al. A preliminary study of volatile agents or total intravenous anesthesia for neurophysiological monitoring during posterior spinal fusion in adolescents with idiopathic scoliosis. Spine (Phila Pa 1976). 2014;39(22):E1318-1324.

6. Yoshiga D, Yoshida A, Kitahara S, et al. Perioperative management of a patient with Bernard-Soulier syndrome that has a refractoriness to platelet transfusion. Asian J Oral and Max Surg. 2011;23:102-104.

7. Kostopanagiotou G, Siafaka I, Sikiotis C, Smyrniotis $\mathrm{V}$. Anesthetic and perioperative management of a patient with Bernard-Soulier syndrome. J Clin Anesth. 2004;16(6):458-460.

8. Balci YI, Gozkeser E, Polat A, Gurses M, Kara CO, Herek O. Perioperative management of tonsilloadenoidectomy and circumcision of a patient with Bernard-Soulier syndrome: case report. Blood Coagul Fibrinolysis. 2014;25(8):907-908.

9. Macedo MB, Brito Jde M, Macedo Pda S, Brito JA. Primigravida with Bernard-Soulier Syndrome: a case report. BMC Res Notes. 2015;8:178.

10. Hartman MJ, Caccamese JF, Jr., Bergman SA. Perioperative management of a patient with Bernard-Soulier syndrome for third molar surgery. Oral Surg Oral Med Oral Pathol Oral Radiol Endod. 2007;103(5):626-629.

11. Saade G, Homsi R, Seoud M. Bernard-Soulier syndrome in pregnancy; a report of four pregnancies in one patient, and review of the literature. Eur J Obstet Gynecol Reprod Biol. 1991;40(2):149-152.

12. Bilal RH, Moideen I, Pyke L, Makahleh Z, FernandezJimenez P, Hasan R. Off-pump coronary artery bypass grafting in a patient with Bernard-Soulier syndrome. Ann Thorac Surg. 2010;90(1):284-285.

13. Tefre KL, Ingerslev J, Sorensen B. Clinical benefit of recombinant factor VIIa in management of bleeds and surgery in two brothers suffering from the Bernard-Soulier syndrome. Haemophilia. 2009;15(1):281-284.
14. Hacihanefioglu A, Tarkun P, Gonullu E. Use of recombinant factor VIIa in the management and prophylaxis of bleeding episodes in two patients with Bernard-Soulier syndrome. Thromb Res. 2007;120(3):455-457.

15. Yuksel O, Koklu S, Ucar E, Sasmaz N, Sahin B. Severe recurrent gastrointestinal bleeding due to angiodysplasia in a Bernard-Soulier patient: an onerous medical concomitance. Dig Dis Sci. 2004;49(5):885-887.

16. Nilsson IM. Clinical pharmacology of aminocaproic and tranexamic acids. J Clin Pathol Suppl (R Coll Pathol). 1980;14:41-47.

17. Du Y, Feng C. The Efficacy of Tranexamic Acid on Blood Loss from Lumbar Spinal Fusion Surgery: A Meta-Analysis of Randomized Controlled Trials. World Neurosurg. 2018.

18. Tobias JD. Strategies for minimizing blood loss in orthopedic surgery. Semin Hematol. 2004;41(1 Suppl 1):145156.

19. Soslau G, Horrow J, Brodsky I. Effect of tranexamic acid on platelet ADP during extracorporeal circulation. Am J Hematol. 1991;38(2):113-119.

20. Weber CF, Gorlinger K, Byhahn C, Moritz A, Hanke AA, Zacharowski K, Meininger D. Tranexamic acid partially improves platelet function in patients treated with dual antiplatelet therapy. Eur J Anaesthesiol. 2011;28(1):5762.

21. Sabovic M, Zupan IP, Salobir B, Zupan I, Cernelc P, Lavre J, Vujkovac B. The effect of long-term, low-dose tranexamic acid treatment on platelet dysfunction and haemoglobin levels in haemodialysis patients. Thromb Haemost. 2005;94(6):1245-1250.

22. Tobias JD. Recombinant factor VIIa in pediatric patients. Trans Alter Trans Med. 2003:5(suppl):40-45.

23. Dutta TK, Verma SP. Rational use of recombinant factor VIIa in clinical practice. Indian J Hematol Blood Transfus. 2014;30(2):85-90.

24. Mackman N, Tilley RE, Key NS. Role of the extrinsic pathway of blood coagulation in hemostasis and thrombosis. Arterioscler Thromb Vasc Biol. 2007;27(8):16871693.

25. Kjalke M, Ezban M, Monroe DM, Hoffman M, Roberts HR, Hedner U. High-dose factor VIIa increases initial thrombin generation and mediates faster platelet activation in thrombocytopenia-like conditions in a cell-based model system. Br J Haematol. 2001;114(1):114-120.

26. Monroe DM, Hoffman M, Roberts HR. Platelets and thrombin generation. Arterioscler Thromb Vasc Biol. 2002;22(9):1381-1389.

27. Almeida AM, Khair K, Hann I, Liesner R. The use of recombinant factor VIIa in children with inherited platelet function disorders. Br J Haematol. 2003;121(3):477-481.

28. Whiting D, DiNardo JA. TEG and ROTEM: technology and clinical applications. Am J Hematol. 2014;89(2):228232.

29. Abdelfattah K, Cripps MW. Thromboelastography and rotational thromboelastometry use in trauma. Int J Surg. 2016;33(Pt B):196-201. 